\title{
Observation of Superradiation (according to R. H. Dicke) in the Nitrogen Oxidation Chain Reaction
}

\section{Abstract}

The superradiation can hypothetically produce parallel and coherent light beams characterized with some phase shift. Radiation with such properties is observed from nitrogen oxidation chain reaction initiated by electric discharge. The superradiating molecules are identified to be $\mathrm{NO}\left(\mathrm{B}^{2} \Pi\right)$

Keywords: superradiation, coherence, interference, phase shift, Electronic Energy Explosion, nitrogen oxidation, chain reaction, electronically excited particles, spectra, $\mathrm{NO}\left(\mathrm{B}^{2} \Pi\right), \mathrm{O}\left({ }^{1} \mathrm{~S}\right)$.
Volume 2 Issue 3 - 2018

\section{Fedotov VG, Fedotova EY}

Semenov Institute of Chemical Physics of Russ Acad of Sciences, Russia

Correspondence: Fedotov VG, Senior Researcher, Semenov Institute of Chemical Physics of Russ Acad of Sciences, Kosygina street, 4, Moscow, I I7977, Russia, Tel 8-(495)- 5649763, Email vgfedotov47@inbox.ru

Received: May 15, 2018 | Published: May 23, 2018

\section{Introduction}

According to R. H. Dicke ${ }^{1}$ an ensemble of electronically excited particles can produce superradiation - coherent spontaneous radiation. The main properties of superradiation are: 1) it is several order of magnitude more intensive then spontaneous radiation; 2) it is coherent; 3 ) its spectrum contains some elements (not all) of spontaneous radiation spectrum. 4) The delay time $\left(\tau_{0}\right)$ between superradiation pulse and the moment of excited molecules formation is much more then correlation time $\left(\tau_{c}\right):\left(\tau_{0}\right) \sim \tau_{c} \ln N$. Is it possible for this reason that two different parts of active zone produce two light beams coherent and parallel but characterized by different phase angle? Interference of these beams in the focal plane of the camera objective lens would give the light spot displaced from the point of interference maximum in the case of zero phase shifts. Electronically excited atoms $\mathrm{O}\left({ }^{1} \mathrm{~S}\right)$ are needed for chain propagation in the endothermic nitrogen oxidation chain reaction. ${ }^{2}$ Such atoms are produced in the mentioned above reaction along with electronically-excited molecules $\mathrm{NO}\left(\mathrm{B}^{2} \Pi\right), \mathrm{NO}_{2}{ }^{*}$ and $\mathrm{N}_{2}-\mathrm{O}\left({ }^{1} \mathrm{~S}\right) .{ }^{2}$ But for the initiation of nitrogen oxidation chain reaction in air the electric discharge is needed, which produces initial concentration of active particles and $\mathrm{NO}$ at minimal heating of the reaction mixture. This condition is satisfied in three cases: 1) pulse-periodic spark discharge with frequency $1000 \mathrm{~Hz}$; 2 pulse discharge near ferrite surface; 3 ) electro-explosion of moistened nitrate (sodium, potassium, calcium and ammonia). In all mentioned above cases is observed stimulated emission of radiation, ${ }^{3-5}$ but in addition to that some phenomena are observed too, which cannot be explained with assumption about super luminescence, but are pointing to superradiation. So one can say, that superradiation is a "visit card of EEE" (Electronic Energy Explosion) in air, producing nitrogen oxidation chain reaction, which is the common mechanism of three mentioned above processes. The aim of present work is the presentation of experimental data which are pointing to superradiation (according to Dicke R. H. ${ }^{1}$ ) and its discussion.

\section{Methods and Results}

Digital camera Sony DSC-650 was used for registering of EEE radiation. Initiation of EEE and nitrate salt electro explosion was described previously. ${ }^{5,6}$ The video of Electronic Energy Explosion (EEE) in the Air initiated by electric discharge near the ferrite surface contains two frames showing the presence of some radiation from the discharge zone. At one of them the intensity of radiation is extremely high, so that whole of frame looks white and only few details of experimental installation can be seen. The before going frame contains only one light spot (of violet color) at the unexpected place: much lower then the place, where the luminous cloud of EEE was located (between the electrodes, touching the ferrite) (Figure 1). Two electrodes are touching the ferrite core used for TV fly back transformers. These electrodes are connected to capacitor battery, charged to $400 \mathrm{~V}$. Two white arrows are pointing to light spot registered at the moment of touching. One arrow points to the point of touching, where the luminous cloud of EEE was produced Figure 2.

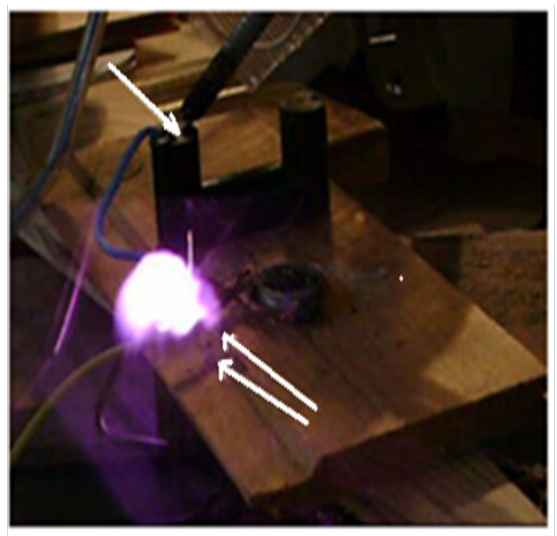

Figure I The video frame, which is foregoing to the frame of highest irradiation. 


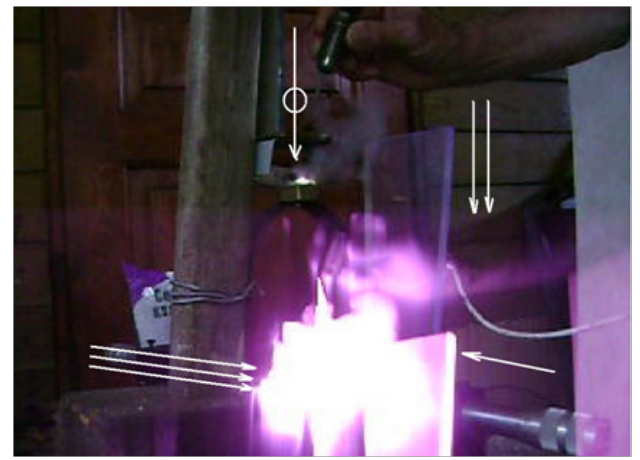

Figure 2 The video frame, which contains the displaced image of luminous cloud (three white arrows) in the case of potassium nitrate electro explosion.

One can see also displaced images of several objects irradiated by the luminous cloud: the peas of organic glass (one white arrow), the operator hand (two white arrows). One white arrow marked with white circle points to the real location of the discharge zone.

\section{The spectrum of superradiator}

Two white arrows are pointing to the center of spectral grating (the expected position of spectrum was here) (Figure 3).

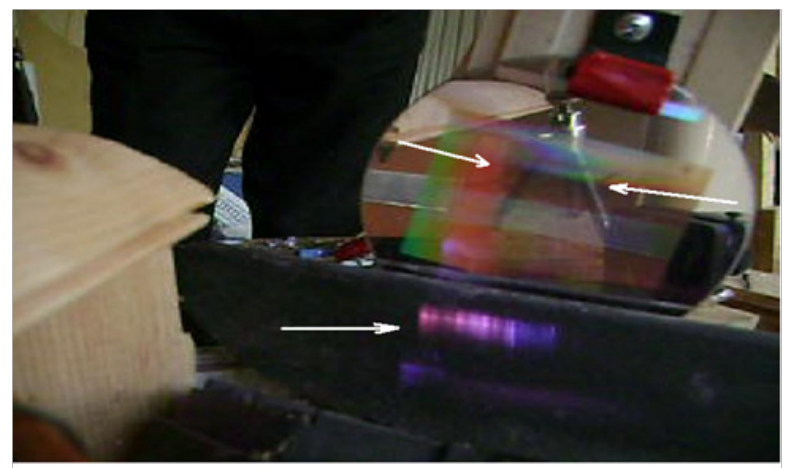

Figure 3 The Spectrum (displaced downward in relation to expected position), one white arrow points to it.
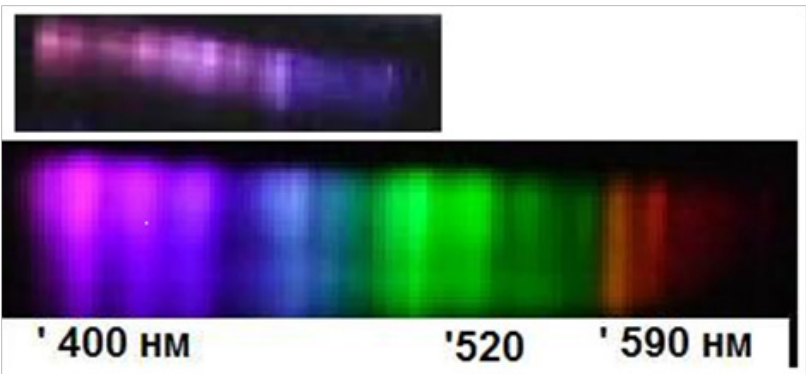

Figure 4 The spectrum of superradiator is placed over the spectrum of NO (B ${ }^{2}$ П)-molecules. ${ }^{6}$

\section{Discussion}

There is no doubt, that photo camera is functioning correctly: images of all subjects irradiated by any light sources (but nitrogen chain reaction at pre EEE stage) are positioned correctly. Our assumption that displaced images on Figure 1 and Figure 2 are resulting from superradiation is based on following observations: 1) at real location of the radiating zone on video frames no radiation can be seen. It means that intensity of spontaneous radiation of excited particles is several orders of magnitude weaker then observed "displacing" radiation. 2) It can be stated, that superradiation on Fig. 2 propagated isotropic in all directions: to the operator hand (to rights), to the peas of organic glass (downward), to the operator fingers (upward). So the displaced images are produced by some isotropic radiation. 3) the displacement of observed images in relation to expected positions (according to laws of geometric optics) indicates on some phase composition of light beams, which are able to interfere (thus - on coherence of that radiation). All mentioned above properties can be ascribed to superradiation. To our knowledge this is the first instant when superradiation resulting from chemical reaction is observed. What can be said about the nature of superradiator? Dark intervals in spectrum of superradiator are positioned similarly to that of the violet part of $\mathrm{NO}\left(\mathrm{B}^{2} \Pi\right)$-molecules spectrum (Figure 4). The fine structure of these spectra is some different. That difference can be combined presumably with superradiation mechanism. So we conclude that observed superradiation is generated by $\mathrm{NO}\left(\mathrm{B}^{2} \Pi\right)$ molecules.

\section{Acknowledgements}

None.

\section{Conflict of interest}

The author declares there is no conflict of interest.

\section{References}

1. Dicke RH. Coherence in spontaneous radiation processes. Phys Rev. 1954;93:99.

2. Fedotov VG, Fedotova E Ya. Chemical Kinetic Model of the Chain Reaction of Atmospheric Nitrogen Oxidation Initiated by Electric Discharge. Russian J of Phys Chem B. 2016;10(5):748-752.

3. Fedotov VG, Fedotova E Ya. The inversed Populations in the active Medium of an Electronic Energy Explosion in the Air. Journal of Physics \& Astronomy. 2017;5(1):106.

4. Fedotov VG, Fedotova E Ya. Multicolor Laser Generation resulting from a Chain reaction of Atmospheric nitrogen oxidation initiated by an Electric Discharge in Air. Russian Journal of Physical Chemistry B. 2017;11(6):928.

5. Fedotov VG, Fedotova E Ya. Observation of Chemiluminescence, Superluminescence, and Light Amplification during Spark-Discharge initiated Thermal Decomposition of Sodium Nitrate. Doklady Physical Chemistry. 2012; 444(2):96-99.

6. Fedotov VG, Fedotova E Ya. Explosion in Atmospheric Air Initiated by an Electric Discharge and Associated with the Growth of the Concentrations of Electronically Excited Species and Free Charges. Russian J Phys Chem B. 2015;9(2):223-227. 